\title{
Clinicopathologic Characteristics of Young Gastric Cancer Patients: Diagnostic Staging Accuracy and Survival
}

\author{
Woochul Kim, M.D. ${ }^{1}$, Sangil Youn, M.D. ${ }^{1}$, Yongjoon Won, M.D. ${ }^{1}$, Sahong Min, M.D. ${ }^{1}$, Young Suk Park, M.D. ${ }^{1}$, \\ Sang-Hoon Ahn, M.D. ${ }^{1}$, Do Joong Park, M.D., Ph.D. ${ }^{2,3}$, Hyung-Ho Kim, M.D., Ph.D. ${ }^{1,2}$ \\ 'Department of Surgery, Seoul National University Bundang Hospital, Seongnam, '2Department of Surgery, Seoul National University College of Medicine, \\ Seoul, ${ }^{3}$ Department of Surgery, Seoul National University Hospital, Seoul, Korea
}

Purpose: The purpose of this study was to investigate the clinicopathologic characteristics of young gastric cancer patients and analyze the risk factors for stage underestimation and survival.

Methods: Relevant data of 5029 patients who underwent surgery for gastric cancer at Seoul National University Bundang Hospital between 2003 to 2014 were collected. Patients were divided based on age (younger group and older group). Clinical stages were compared to pathologic stages for accuracy, and risk factors for underestimation were analyzed using univariate and multivariate analysis regression. Overall survival and cancer-specific survival were analyzed using the Kaplan-Meier method.

Results: A total of 4396 patients were eligible for inclusion. The younger group was an independent risk factor for nodal metastasis ( $\mathrm{RR}=1.44,95 \% \mathrm{CI} 1.06 \sim 1.95)$ and an independent risk factor for clinical N-stage underestimation $(\mathrm{RR}=1.50,95 \% \mathrm{CI}=1.14 \sim 1.98)$. However, there was no significant difference in 5 -year cancer-specific survival for both age groups $(92.2 \%$ vs $90.2 \%, p=0.306)$.

Conclusion: In conclusion, intra-operative investigation of T-stage with standard operation should be done in young gastric cancer patients as they have a higher incidence of lymph node metastasis, with greater frequency of stage underestimation.

Keywords: Stomach neoplasm, Diagnosis, Survival, Age groups, Surgery

Supplementary file: This article contains supplementary material (https://doi.org/10.7602/ jmis.2020.23.4.163).

This is an Open Access article distributed under the terms of the Creative Commons Attribution Non-Commercial License (http:// creativecommons.org/licenses/by-nc/4.0/) which permits unrestricted non-commercial use, distribution, and reproduction in any medium, provided the original work is properly cited.
Received July 30, 2020

Revised September 29, 2020

Accepted October 30, 2020

Corresponding author

Young Suk Park

Department of Surgery, Seoul

National University Bundang

Hospital, 166 Gumi-ro, Bundang-gu, Seongnam 13620, Korea

Tel: $+82-31-787-7099$

Fax: +82-31-787-4055

E-mail: youngsukmd@gmail.com

ORCID:

https://orcid.org/0000-0002-6352-9759
Copyright (c) 2020 The Journal of Minimally Invasive Surgery. All rights reserved.

\section{INTRODUCTION}

Gastric cancer is the 5th most common cancer and 3rd most common cause of cancer-related deaths worldwide, ${ }^{1}$ with incidence rates highly concentrated in East Asia and Latin America. ${ }^{1,2}$ Recent advances in surgical and endoscopic diagnostic techniques have progressively improved patient outcomes, becoming vitally important for establishing accurate clinical staging and determining suitable treatment strategies.
There are multiple challenges in making accurate clinical diagnosis for gastric cancer; one of which is accurately examining the lymph node metastasis. Based on previous studies, diffuse type cancers have more lymph node metastasis compared with its counterparts, ${ }^{3}$ and they are often inaccurately diagnosed. ${ }^{4,5}$ Underestimation of lymph node metastasis at the pre-operative stage may pose as an obstacle in deciding the correct range of lymph node dissection. Since young patients are known to have higher frequencies of undifferentiated and diffuse tumor 
types, ${ }^{6-8}$ it is even more challenging to appropriately determine treatment strategies.

Hence, the purpose of this study was twofold: (1) to investigate the accuracy of clinical staging based on two - younger and older - age groups; and (2) to evaluate whether the underestimation of pre-operative clinical staging is more prevalent in the younger population than in the older population. We hypothesized that it would be more prevalent in the younger population compared with the older population, and thus, influencing survival.

\section{MATERIALS AND METHODS}

\section{Patients}

A total of 5029 patients who underwent radical gastrectomy for gastric cancer between May 1st 2003 and December 31st 2014 at Seoul National University Bundang Hospital were initially screened. Patients who underwent non-curative surgery (381), non-radical gastrectomy (183) without pathologic data (59), or with multiple gastric cancers (10) were excluded from this study because they may hinder a clear evaluation of the surgical outcomes. A total of 4396 patients were eligible for investigation. This study was approved by the Institutional Review Board and was performed in accordance with the principles of the Declaration of Helsinki. The requirement for informed consent was waived due to the retrospective nature of this study, as data were de-identified prior to analysis.

\section{Data collection and definition of variables}

This study retrospectively analyzed the patient records for name, patient number, stage, gender, body mass index (BMI), American Society of Anesthesiologists (ASA) physical status class, tumor location (esophagogastric junction, proximal, middle, distal), average tumor size, Lauren histologic classification (intestinal, diffuse, mixed), operation name, operation time, estimated blood loss (EBL), clinical stage, pathologic stage, and survival. Tumors were staged according to the American Joint Committee on Cancer (AJCC), 8th edition. Clinical staging was evaluated using the pre-operative computed tomography (CT) and endoscopic ultrasonography (EUS) from the patient medical records. Clinical stage was considered conservatively; higher stage of the two exams were selected. Patients without EUS results and no visualization of the main tumor in CT were designated as T1. Accuracy, overestimation, and underestimation of clinical staging was determined by comparing the clinical staging to the pathologic staging. Identical pathologic stage was designated as accurate; lower pathologic staging was designated as overestimation; and higher pathologic staging was designated as underestimation.
Patients' death information was obtained from the microdata integrated service database of the Korea Statistics Promotion Institute. Recurrence data was collected through reviewing the medical record for follow-up CT scans, esophagoduodenoscopy (EGD), or ultrasonography. Abdominal ultrasonography and computed tomography (CT) were checked every 6 months in the case of early gastric cancer patients, and abdomen-pelvis CT scan was evaluated least every 6 months in advanced cancer patients. The survival and recurrence statuses were determined in March 2020.

According to recently nationwide statistics in Korea, there is a demographic differences for age below $40,{ }^{9}$ and clinicopathologic features analyzed in this study tended to be different between patients aged 40 years or less and those aged 40 years or over. Thus, we divided our study population into two groups based on age cut-off of 40 years of age. This is similar to cut-off age used in previous studies. ${ }^{7,10,11}$

\section{Statistical analysis}

Categorical data were compared using Pearson's Chi-squared test, and continuous data were compared using the independent $\mathrm{t}$ test. Categorical variables were presented as numbers with percentages, and continuous data were expressed as the mean \pm standard deviation. Survival was examined using the Kaplan Meier method. A $p$ value of less than 0.05 was considered to be statistically significant. Data analysis was done using the SPSS program version 22 (SPSS, Inc., Chicago, IL, USA).

\section{RESULTS}

\section{Baseline characteristics}

All patients underwent $\mathrm{R} 0$ resection of tumor with radical lymph node dissection. The median follow-up was 52.2 months after surgery. Demographics of the younger group (YG) and the older group (OG) are outlined in Table 1. Female was notably more prominent in the younger group compared with the older group. There was no significant difference in the number patients within each pathologi T-staging between the two groups; both groups showed a higher proportion of the $\mathrm{T} 1$ stage $(62.6 \%$ in YG vs. 60.2 in OG). Similar trend was found for the pathologic $\mathrm{N}$ stage, where N0 had the highest proportion in both groups. The younger group had a higher incidence of lymph node invasion compared with the older group (41.2\% vs. $34.9 \%$ ). TNM stage was similar in both groups, with no significant statistical difference. A higher proportion of diffuse or mixed type cancers was seen in the younger group than in the older group (86.6\% vs. 44.2 ). As expected, the younger group had a significantly lower ASA class than the older group; however, the mean BMI was similar 
Table 1. Patient characteristics

\begin{tabular}{|c|c|c|}
\hline & Young (N=337, \%) & Old ( $\mathrm{N}=4059, \%)$ \\
\hline Age & 35.99 (20 40) & 61.98 (41 92) \\
\hline \multicolumn{3}{|l|}{ Gender (\%) } \\
\hline M & $156(46.3)$ & 2792 (68.8) \\
\hline $\mathrm{F}$ & $181(53.7)$ & 1267 (31.2) \\
\hline \multicolumn{3}{|l|}{ Location (\%) } \\
\hline GE Junction & $5(1.5)$ & $85(2.1)$ \\
\hline Proximal & $59(17.5)$ & $679(16.7)$ \\
\hline Middle & $123(36.5)$ & $943(23.2)$ \\
\hline Distal & $137(40.7)$ & 2269 (55.9) \\
\hline Diffuse & $13(3.9)$ & $83(2.0)$ \\
\hline Average tumor size (cm) & $3.918(0.4 \sim 19.0)$ & $3.891(0.1 \sim 21.0)$ \\
\hline \multicolumn{3}{|l|}{ Clinical stage (\%) } \\
\hline $\mathrm{T} 1$ & $185(54.9)$ & $2064(50.8)$ \\
\hline T2 & $77(22.8)$ & $845(20.8)$ \\
\hline T3 & $56(16.6)$ & $819(20.2)$ \\
\hline T4 & $19(5.6)$ & 331 (8.2) \\
\hline NO & $264(78.3)$ & $3036(74.8)$ \\
\hline N1 & $64(19.0)$ & $820(20.2)$ \\
\hline N2 & $6(1.8)$ & $159(3.9)$ \\
\hline N3 & $3(0.9)$ & $44(1.1)$ \\
\hline \multicolumn{3}{|l|}{ Pathologic stage (\%) } \\
\hline $\mathrm{T} 1$ & $211(62.6)$ & $2442(60.2)$ \\
\hline T2 & $25(7.4)$ & 499 (12.3) \\
\hline T3 & $59(17.5)$ & $632(15.6)$ \\
\hline T4 & $42(12.5)$ & $486(12.0)$ \\
\hline NO & $198(58.8)$ & 2649 (65.3) \\
\hline N1 & $49(14.5)$ & 494 (12.3) \\
\hline N2 & $54(16.0)$ & $370(9.1)$ \\
\hline N3 & $36(10.7)$ & $546(13.5)$ \\
\hline \multicolumn{3}{|l|}{ TNM } \\
\hline Stage I & $204(60.5)$ & $2616(64.4)$ \\
\hline Stage \| & $53(15.7)$ & $625(15.4)$ \\
\hline Stage III & $80(23.7)$ & $818(20.2)$ \\
\hline \multicolumn{3}{|l|}{ Histology (\%) } \\
\hline Intestinal & 45 (13.4) & 2268 (55.9) \\
\hline Diffuse & $274(81.3)$ & 1590 (39.2) \\
\hline Mixed & $18(5.3)$ & $201(5.0)$ \\
\hline $\mathrm{BMI}\left(\mathrm{kg} / \mathrm{m}^{2}\right)$ & 22.54 (14.43 34.60) & 23.67 (14.01 37.26) \\
\hline
\end{tabular}


Table 1. Continued

\begin{tabular}{|c|c|c|}
\hline & Young $(\mathrm{N}=337, \%)$ & Old $(\mathrm{N}=4059, \%)$ \\
\hline \multicolumn{3}{|l|}{ ASA class (\%) } \\
\hline 1 & $288(85.5)$ & $1902(46.9)$ \\
\hline 2 & $47(13.9)$ & $1951(48.1)$ \\
\hline$\geq 3$ & $2(0.6)$ & $206(5.0)$ \\
\hline \multicolumn{3}{|l|}{ Operation approach (\%) } \\
\hline Laparoscopic & $262(77.7)$ & $2886(71.1)$ \\
\hline Open & $9(2.7)$ & $91(2.2)$ \\
\hline Open Conversion & $66(19.6)$ & $1082(26.7)$ \\
\hline \multicolumn{3}{|l|}{ Type of operation (\%) } \\
\hline Distal gastrectomy & $255(75.7)$ & $3181(78.4)$ \\
\hline Total gastrectomy & $65(19.3)$ & $676(16.7)$ \\
\hline Proximal gastrectomy & $13(3.9)$ & $175(4.3)$ \\
\hline Pylorus preserving gastrectomy & $4(1.2)$ & $27(0.7)$ \\
\hline \multicolumn{3}{|l|}{ Lymph Node Dissection } \\
\hline $\mathrm{D} 1+$ & $131(38.9)$ & $1523(37.5)$ \\
\hline$\geq \mathrm{D} 2$ & $206(61.1)$ & $2536(62.5)$ \\
\hline Retrieved Lymph Nodes & $51.84(18 \sim 115)$ & $51.19(6 \sim 221)$ \\
\hline Positive Lymph Nodes & $2.70(0 \sim 41)$ & $2.89(0 \sim 104)$ \\
\hline Op Time (min) & 183.33 (70 495) & 185.18 (55 720) \\
\hline $\mathrm{EBL}(\mathrm{mL})$ & $102.97(10 \sim 1200)$ & 123.29 (10 6650) \\
\hline Hospital stay (days) & $6.93(4 \sim 41)$ & 8.43 (2 1432) \\
\hline
\end{tabular}

Table 2. Tendency of node metastasis for clinical and pathologic T stage

\begin{tabular}{|c|c|c|c|}
\hline & Pathologic node negative & Pathological node positive & $p$ value \\
\hline Clinical early gastric cancer (cT1) & & & 0.007 \\
\hline Younger group & $146(78.9)$ & $39(21.1)$ & \\
\hline Older group & 1780 (86.2) & $284(13.8)$ & \\
\hline Clinical advanced gastric cancer ( $\geq c T 2)$ & & & 0.025 \\
\hline Younger group & $52(34.2)$ & 100 (65.8) & \\
\hline Older group & $869(43.6)$ & $1126(56.4)$ & \\
\hline Pathologic early gastric cancer (pT1) & & & 0.011 \\
\hline Younger group & $173(82.0)$ & 38 (18.0) & \\
\hline Older group & $2149(88.0)$ & $293(12.0)$ & \\
\hline Pathologic advanced gastric cancer ( $\geq p T 2)$ & & & 0.001 \\
\hline Younger group & 25 (19.8) & $101(80.2)$ & \\
\hline Older group & $500(30.9)$ & $1117(69.1)$ & \\
\hline
\end{tabular}

in both groups. Both the younger group and the older group had similar operations, with most patients receiving the laparoscopic approach. The younger group had more laparoscopic approaches than the older group (77.7\% vs. $71.1 \%)$, and lower open conversion surgeries (19.6\% vs. $26.7 \%)$. Most patients underwent distal gastrectomy (75.7\% vs. $78.4 \%)$, followed by total gastrectomy (19.3\% 
vs. 16.7), and function preserving gastrectomy (1.2\% vs. $0.7 \%)$. The majority of patients in both groups underwent D2 lymph node dissection (61.1\% vs $62.5 \%$ ), with a similar average of number of retrieved lymph nodes (51.84 vs 51.19) and positive lymph nodes (2.70 vs 2.89 ). Both groups had comparable operation time and estimated blood loss.

\section{Node metastasis according to T-stages}

Node metastasis was more frequently observed in the younger group than in the older group for clinical early gastric cancer (EGC) (21.1\% in YG vs. $13.8 \%$ in OG, $p=0.007)$ as well as pathologic EGC $(18.0 \%$ vs $12.0 \%, p=0.011)$ (Table 2). Furthermore, similar results were seen in both clinical advanced gastric cancer (AGC) (65.8\% vs $56.4 \% p=0.025)$ and pathologic AGC ( $80.2 \%$ vs $69.1 \%$ $p=0.001)$. The younger group was an independent risk factor for nodal metastasis ( $\mathrm{RR}=1.44,95 \% \mathrm{CI} 1.06 \sim 1.95)$, as with large tumor size ( $\mathrm{RR}=2.94,95 \% \mathrm{CI} 2.22 \sim 3.89)$ and higher T-stage and diffuse or mixed type histology ( $\mathrm{RR}=1.25,95 \% \mathrm{CI} 1.06 \sim 1.49)$ (Table 3).

\section{Accuracy of preoperative staging}

Clinical T-stage accuracy in terms of histologic types showed that there was a difference of accuracy, underestimation and overestimation rates between diffuse and mixed types compared with the intestinal type ( $p=0.033$ ) (Table 4$)$. And underestimation rate was higher for the diffuse and mix types compared with the intestinal type (23.2\% vs $10.3 \%)$. Similarly, accuracy, underestimation and overestimation rates showed difference comparing the different age groups $(p<0.001)$ with underestimation rates higher for the younger group compared with the older group $(20.5 \%$ vs $16.1 \%)$. This trend continued when comparing between the

Table 3. Risk factors for lymph node metastasis

\begin{tabular}{|c|c|c|c|c|c|c|}
\hline & \multicolumn{3}{|c|}{ Univariable analysis } & \multicolumn{3}{|c|}{ Multivariable analysis } \\
\hline & Odds ratio & $95 \% \mathrm{Cl}$ & $p$ value & Odds ratio & $95 \% \mathrm{Cl}$ & $p$ value \\
\hline \multicolumn{7}{|l|}{ Age } \\
\hline$\geq 40$ years & Reference & & & & & \\
\hline$<40$ years & 1.32 & $1.05 \sim 1.65$ & 0.017 & 1.4 & $1.03 \sim 1.89$ & 0.031 \\
\hline \multicolumn{7}{|l|}{ Gender } \\
\hline Male & Reference & & & & & \\
\hline Female & 1.15 & $1.01 \sim 1.31$ & 0.033 & 1.15 & $0.97 \sim 1.37$ & 0.867 \\
\hline \multicolumn{7}{|l|}{ Tumor size } \\
\hline$<2 \mathrm{~cm}$ & Reference & & & & & \\
\hline$\geq 2 \mathrm{~cm}$ & 10.19 & 7.89 13.18 & $<0.001$ & 3.01 & $2.28 \sim 3.99$ & $<0.001$ \\
\hline \multicolumn{7}{|l|}{ pT-stage } \\
\hline $\mathrm{T} 1$ & Reference & & & & & \\
\hline $\mathrm{T} 2$ & 6.02 & $4.90 \sim 7.40$ & $<0.001$ & 4.99 & $4.02 \sim 6.18$ & $<0.001$ \\
\hline T3 & 17.59 & $14.38 \sim 21.52$ & $<0.001$ & 13.42 & $10.85 \sim 16.61$ & $<0.001$ \\
\hline $\mathrm{T} 4$ & 73.51 & $53.18 \sim 101.60$ & $<0.001$ & 50.84 & $36.3 \sim 71.22$ & $<0.001$ \\
\hline \multicolumn{7}{|l|}{ Tumor location } \\
\hline Distal & Reference & & & & & \\
\hline Middle & 1.14 & $0.98 \sim 1.34$ & 0.087 & 0.79 & $0.65 \sim 0.97$ & 0.024 \\
\hline Upper & 1.91 & $1.62 \sim 2.24$ & $<0.001$ & 0.88 & $0.71 \sim 1.10$ & 0.263 \\
\hline Diffuse & 22.15 & $11.09 \sim 44.24$ & $<0.001$ & 2.01 & $0.90 \sim 4.47$ & 0.089 \\
\hline \multicolumn{7}{|l|}{ Histology } \\
\hline Intestinal & Reference & & & & & \\
\hline Diffuse \& mixed & 2.06 & $1.81 \sim 2.33$ & $<0.001$ & 1.25 & $1.06 \sim 1.49$ & 0.010 \\
\hline
\end{tabular}


Table 4. Accuracy of clinical staging

\begin{tabular}{lccc}
\hline & Accurate & Underestimated & Overestimated \\
\hline T-stage & & & \\
Younger Group & $211(62.6)$ & $69(20.5)$ & $57(16.9)$ \\
Older Group & $2530(62.3)$ & $653(16.1)$ & $876(21.6)$ \\
Intestinal type & $1524(65.9)$ & $238(10.3)$ & $551(23.8)$ \\
Diffuse and mixed type & $1217(58.4)$ & $484(23.2)$ & $382(18.3)$ \\
N-Stage & & & $24(7.1)$ \\
Younger Group & $223(66.2)$ & $90(26.7)$ & $329(8.1)$ \\
Older Group & $3014(74.3)$ & $716(17.6)$ & $189(8.2)$ \\
Intestinal type & $1802(77.9)$ & $322(13.9)$ & $164(7.9)$ \\
Diffuse and mixed type & $1435(69.0)$ & $484(23.2)$ & $<0.001$ \\
\end{tabular}

Table 5. Risk factors for underestimation of T-staging

\begin{tabular}{|c|c|c|c|c|c|c|}
\hline & \multicolumn{3}{|c|}{ Univariable analysis } & \multicolumn{3}{|c|}{ Multivariable analysis } \\
\hline & Odds ratio & $95 \% \mathrm{Cl}$ & $p$ value & Odds ratio & $95 \% \mathrm{Cl}$ & $p$ value \\
\hline \multicolumn{7}{|l|}{ Age } \\
\hline$\geq 40$ years & Reference & & & & & \\
\hline$<40$ years & 1.34 & $1.02 \sim 1.77$ & 0.037 & 0.89 & $0.66 \sim 1.20$ & 0.441 \\
\hline \multicolumn{7}{|l|}{ Gender } \\
\hline Male & Reference & & & & & \\
\hline Female & 1.27 & $1.07 \sim 1.49$ & 0.005 & 1.05 & $0.88 \sim 1.26$ & 0.564 \\
\hline \multicolumn{7}{|l|}{ Tumor size } \\
\hline$<2 \mathrm{~cm}$ & Reference & & & & & \\
\hline$\geq 2 \mathrm{~cm}$ & 7.87 & & $<0.001$ & 6.27 & $4.30 \sim 9.13$ & $<0.001$ \\
\hline \multicolumn{7}{|l|}{ Tumor location } \\
\hline Distal & Reference & & & & & \\
\hline Middle & 2.09 & $1.71 \sim 2.54$ & $<0.001$ & 1.71 & $1.40 \sim 2.10$ & $<0.001$ \\
\hline Upper & 2.47 & $2.01 \sim 3.03$ & $<0.001$ & 2.04 & $1.65 \sim 2.52$ & $<0.001$ \\
\hline Diffuse & 7.78 & $5.11 \sim 11.85$ & $<0.001$ & 4.36 & $2.84 \sim 6.70$ & $<0.001$ \\
\hline \multicolumn{7}{|l|}{ Histology } \\
\hline Intestinal & Reference & & & & & \\
\hline Diffuse and mixed & 2.68 & $2.26 \sim 3.18$ & $<0.001$ & 2.05 & $1.71 \sim 2.46$ & $<0.001$ \\
\hline
\end{tabular}

younger and older groups for the diagnostic accuracy of clinical $\mathrm{N}$-staging.

In observing similar trends for a higher underestimation rate in the clinical staging for both diffuse type tumor and the younger group, we had conducted a subgroup analysis to see whether age played a role in making a difference in diagnostic accuracy rates (Supplementary Table 1). Both groups had no different accuracy rates for clinical T-stage when analyzed separate- ly under each histologic type. Contrastingly, when analyzing the clinical N-stage accuracy for diffuse and mixed types of tumor, the younger group showed difference in diagnostic accuracy compared with the older group.

\section{Risks of clinical stage underestimation}

Table 5 illustrates the risk factors for clinical T-stage under- 
estimation. In a multivariable analysis, tumor size greater than $2 \mathrm{~cm}$ was shown to have a higher relative risk $(\mathrm{RR}=6.27,95 \%$ $\mathrm{CI}=4.30 \sim 9.13)$ compared with those less than $2 \mathrm{~cm}$ in size. No- tably, tumors located in the upper portions of the stomach were shown to have a higher risk of underestimation than those located in the distal portions of the stomach. As expected, diffuse

Table 6. Risk factors for underestimation of N-staging

\begin{tabular}{|c|c|c|c|c|c|c|}
\hline & \multicolumn{3}{|c|}{ Univariable analysis } & \multicolumn{3}{|c|}{ Multivariable analysis } \\
\hline & Odds ratio & $95 \% \mathrm{Cl}$ & $p$ value & Odds ratio & $95 \% \mathrm{Cl}$ & $p$ value \\
\hline \multicolumn{7}{|l|}{ Age } \\
\hline$\geq 40$ years & Reference & & & & & \\
\hline$<40$ years & 1.7 & $1.32 \sim 2.19$ & $<0.001$ & 1.5 & $1.14 \sim 1.98$ & 0.004 \\
\hline \multicolumn{7}{|l|}{ Gender } \\
\hline Male & Reference & & & & & \\
\hline Female & 1.37 & $1.17 \sim 1.61$ & $<0.001$ & 1.27 & $1.07 \sim 1.51$ & 0.006 \\
\hline \multicolumn{7}{|l|}{ Tumor size } \\
\hline$<2 \mathrm{~cm}$ & Reference & & & & & \\
\hline$\geq 2 \mathrm{~cm}$ & 4.95 & $3.70 \sim 6.62$ & $<0.001$ & 2.77 & $2.04 \sim 3.77$ & $<0.001$ \\
\hline \multicolumn{7}{|l|}{ pT-stage } \\
\hline $\mathrm{T} 1$ & Reference & & & & & \\
\hline Т2 & 3.7 & $2.96 \sim 4.63$ & $<0.001$ & 3.11 & $2.46 \sim 3.93$ & $<0.001$ \\
\hline T3 & 3.6 & $2.93 \sim 4.42$ & $<0.001$ & 2.82 & $2.26 \sim 3.51$ & $<0.001$ \\
\hline T4 & 4.14 & $3.32 \sim 5.17$ & $<0.001$ & 3.02 & $2.37 \sim 3.86$ & $<0.001$ \\
\hline \multicolumn{7}{|l|}{ Tumor location } \\
\hline Distal & Reference & & & & & \\
\hline Middle & 1.26 & $1.04 \sim 1.51$ & 0.016 & 1 & $0.82 \sim 1.22$ & 0.981 \\
\hline Upper & 1.25 & $1.02 \sim 1.53$ & 0.03 & 0.84 & $0.68 \sim 1.05$ & 0.118 \\
\hline Diffuse & 2.65 & $1.72 \sim 4.09$ & $<0.001$ & 1 & $0.63 \sim 1.59$ & 0.994 \\
\hline \multicolumn{7}{|l|}{ Histology } \\
\hline Intestinal & Reference & & & & & \\
\hline Diffuse \& Mixed & 1.87 & $1.60 \sim 2.19$ & $<0.001$ & 1.36 & $1.14 \sim 1.62$ & $<0.001$ \\
\hline
\end{tabular}
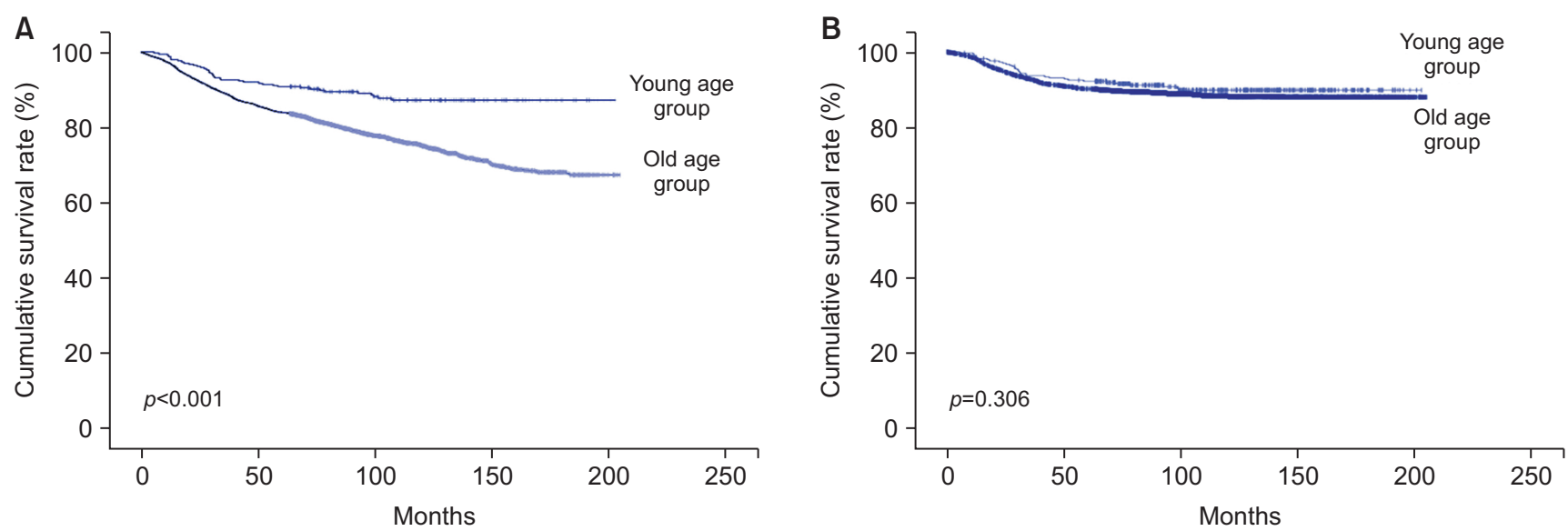

Fig. 1. Survival curves for age groups. (A) Overall survival. (B) Cancer-specific survival. 
and mixed tumor types were shown to have a higher risk of underestimation compared with the intestinal tumor type $(\mathrm{RR}=2.05$, 95\% CI=1.71 2.46).

In contrast to the risks of clinical T-stage underestimation, multivariable analysis revealed a higher risk of clinical N-stage underestimation in both younger age $(\mathrm{RR}=1.50,95 \% \mathrm{CI}=1.14 \sim 1.98)$ and female gender $(\mathrm{RR}=1.27,95 \% \mathrm{CI}=1.07 \sim 1.51)$ (Table 6). Larger tumor size and higher pathologic T-stage also showed a higher risk of nodal stage underestimation. As anticipated, diffuse and mixed tumor types were likely to have a higher risk of nodal underestimation $(\mathrm{RR}=1.36,95 \% \mathrm{CI}=1.14 \sim 1.62)$. unlike T-staging, location of tumor did not have an impact in nodal stage underestimation.

\section{Overall survival and cancer specific survival}

The median follow-up periods were 111.1 months for the younger group and 101.8 months for the older group. The overall 5 -year survival for the older group was significantly lower than that for the younger group ( $90.8 \%$ vs $84.0 \%, p<0.001)$ (Fig. 1A). There was no significant difference in 5 -year cancer-specific survival for both groups (92.2\% vs $90.2 \%, p=0.306$ ) (Fig. 1B). Recurrence pattern showed no difference in both age groups (Supplementary Table 2).

\section{DISCUSSION}

Similar to previous studies, a higher proportion of females and a higher proportion of diffuse cancer type were seen in the younger population. ${ }^{12-14}$ It still remains unclear why females are more prevalent in the younger population with gastric cancer. Some studies have hypothesized that estrogen may have an impact in gastric cancer growth, ${ }^{10,15,16}$ as estrogen receptors were found in some gastric cancer patients. Whether or not estrogen may stimulate cancer growth, its association with the diffuse cancer type is convincing. ${ }^{1718}$

More laparoscopic surgeries are performed in younger patients. This is because older patients are likely to have past surgical histories or other comorbidities that may come as an obstacle in laparoscopic surgeries. That is also the reason why open conversion surgeries are also more frequent among older patients; laparoscopic approach performed in higher stages of gastric cancer may require longer duration of surgery, which may jeopardize post-operative recovery.

Previous studies have revealed that undifferentiated carcinoma is more prevalent in younger patients and that undifferentiated tumors usually grow vertically. ${ }^{19}$ Furthermore, CT scans have an accuracy rate of $60 \sim 70 \%,{ }^{4,5,20}$ when it comes to the evaluation of pre-operative nodal staging with a risk of nodal metastasis based on tumor depth instead of histologic subtypes. ${ }^{21}$ These may explain the underestimation of the T-stage and N-stage in the younger group. Therefore, a thorough intra-operative investigation for enlarged lymph nodes is warranted in younger patients.

Herein, similar to previous studies, the overall survival turned out to be better for the younger group. ${ }^{12-14}$ However, there was no difference in cancer specific survival between the two group. Initially, we assumed that since underestimation of nodal metastasis was higher in the younger group, cancer specific survival would be worse for the younger group, since the scope of surgical dissection is usually determined by the pre-operative staging. Similar cancer specific survival may be attributable to the fact that we have performed more radical lymph node dissection than expected. As an example, in the younger group, the number of EGC patients with node metastasis was higher (the younger group, $18.0 \%$ vs the older group, $12.0 \%$ ), but the number of patients who had D2 or higher lymph node dissection was lower (YG $47.9 \%$ vs $48.5 \%$ ). Similar trend was also observed in AGC patients. Lymph node metastasis were seen in $80.2 \%$ of the younger group and $69.1 \%$ of the older group for AGC patients, with number of patients who had D2 or higher lymph node dissection were $83.4 \%$ for the younger group and $83.8 \%$ for the older group. Since more radical surgery was performed regardless of the pre-operative staging, the influence of lymph node dissection became a negligible factor.

In conclusion, intra-operative investigation of T-stage with standard operation should be done in young gastric cancer patients as they have a higher incidence of lymph node metastasis, with greater frequency of stage underestimation.

\section{ORCID}

Woochul Kim, https://orcid.org/0000-0003-0615-0563

Sangil Youn, https://orcid.org/0000-0002-4029-8572

Yongjoon Won, https://orcid.org/0000-0001-9222-288X

Sahong Min, https://orcid.org/0000-0002-6150-7935

Young Suk Park, https://orcid.org/0000-0002-6352-9759

Sang-Hoon Ahn, https://orcid.org/0000-0001-8827-3625

Do Joong Park, https://orcid.org/0000-0001-9644-6127

Hyung-Ho Kim, https://orcid.org/0000-0002-8916-0048

\section{AUTHORS' CONTRIBUTIONS}

Conceptualization: Woochul Kim, Sangil Youn, Yongjoon Won, Sahong Min, Young Suk Park, Sang-Hoon Ahn, Do Joong Park, and Hyung-Ho Kim. Formal analysis: Woochul Kim, Sangil Youn, Yongjoon Won, Sahong Min, Young Suk Park, Sang-Hoon Ahn, Do Joong Park, and Hyung-Ho Kim. Methodology: Woochul Kim, Sangil Youn, Yongjoon Won, Sahong Min, Young Suk Park, Sang-Hoon Ahn, Do Joong Park, and HyungHo Kim. Writing-original draft: Woochul Kim and Young Suk 
Park. Writing-review and editing: Woochul Kim and Young Suk Park.

\section{CONFLICT OF INTEREST}

None.

\section{FUNDING}

None.

\section{ACKNOWLEDGMENTS}

None.

\section{REFERENCES}

1) Rawla P, Barsouk A. Epidemiology of gastric cancer: global trends, risk factors and prevention. Prz Gastroenterol 2019;14:26-38.

2) Bray F, Ferlay J, Soerjomataram I, Siegel RL, Torre LA, Jemal A. Global cancer statistics 2018: GLOBOCAN estimates of incidence and mortality worldwide for 36 cancers in 185 countries. CA Cancer J Clin 2018;68:394-424.

3) Pyo JH, Lee $\mathrm{H}$, Min BH, et al. Early gastric cancer with a mixed-type Lauren classification is more aggressive and exhibits greater lymph node metastasis. J Gastroenterol 2017;52:594-601.

4) Kim AY, Kim HJ, Ha HK. Gastric cancer by multidetector row CT: preoperative staging. Abdom Imaging 2005;30:465-472.

5) Habermann CR, Weiss F, Riecken R, et al. Preoperative staging of gastric adenocarcinoma: comparison of helical CT and endoscopic US. Radiology 2004;230:465-471.

6) Eom BW, Jung KW, Won YJ, Kim YW. Trends and Outcomes of Non-compliance with Treatment for Gastric Cancer in Korea over the 16 years from 1999 to 2015. J Gastric Cancer 2019;19:92-101.

7) Saito H, Takaya S, Fukumoto Y, Osaki T, Tatebe S, Ikeguchi M. Clinicopathologic characteristics and prognosis of gastric cancer in young patients. Yonago Acta Med 2012;55:57-61.

8) Park HJ, Ahn JY, Jung HY, et al. Clinical characteristics and out- comes for gastric cancer patients aged 18-30 years. Gastric Cancer 2014;17:649-660

9) Ko K-P. Epidemiology of gastric cancer in Korea. J Korean Med Assoc 2019;62:398-406

10) Isobe $T$, Hashimoto $K$, Kizaki J, et al. Characteristics and prognosis of gastric cancer in young patients. Oncol Rep 2013;30:43-49.

11) Wang Z, Xu J, Shi Z, et al. Clinicopathologic characteristics and prognostic of gastric cancer in young patients. Scand J Gastroenterol 2016; 51:1043-1049.

12) Kim DY, Joo JK, Ryu SY, Park YK, Kim YJ, Kim SK. Clinicopathologic characteristics of gastric carcinoma in elderly patients: a comparison with young patients. World J Gastroenterol 2005;11:22-26.

13) Liu S, Feng $\mathrm{F}, \mathrm{Xu} \mathrm{G}$, et al. Clinicopathological features and prognosis of gastric cancer in young patients. BMC Cancer 2016;16:478.

14) Choi JH, Chung $\mathrm{HC}$, Yoo NC, et al. Gastric cancer in young patients who underwent curative resection. Comparative study with older patients. Am J Clin Oncol 1996;19:45-48.

15) Derakhshan MH, Liptrot S, Paul J, Brown IL, Morrison D, McColl KE. Oesophageal and gastric intestinal-type adenocarcinomas show the same male predominance due to a 17 year delayed development in females. Gut 2009;58:16-23.

16) Ebert MP, Malfertheiner P. Review article: Pathogenesis of sporadic and familial gastric cancer--implications for clinical management and cancer prevention. Aliment Pharmacol Ther 2002;16:1059-1066.

17) Tokunaga A, Nishi K, Matsukura N, et al. Estrogen and progesterone receptors in gastric cancer. Cancer 1986;57:1376-1379.

18) Chandanos E, Rubio CA, Lindblad M, et al. Endogenous estrogen exposure in relation to distribution of histological type and estrogen receptors in gastric adenocarcinoma. Gastric Cancer 2008;11:168-174.

19) Takatsu $Y$, Hiki N, Nunobe $S$, et al. Clinicopathological features of gastric cancer in young patients. Gastric Cancer 2016;19:472-478.

20) Fukuya $T$, Honda $H$, Hayashi $T$, et al. Lymph-node metastases: efficacy for detection with helical CT in patients with gastric cancer. Radiology 1995;197:705-711.

21) Lee JH, Choi IJ, Kook MC, Nam BH, Kim YW, Ryu KW. Risk factors for lymph node metastasis in patients with early gastric cancer and signet ring cell histology. Br J Surg 2010;97:732-736. 